\title{
Meditative Movement for Respiratory Function: A Systematic Review
}

\author{
Ava B Lorenc PhD, Yuyi Wang, Susan L Madge PhD, Xiaoyang Hu MSc, \\ Awais M Mian, and Nicola Robinson PhD
}

\author{
Introduction \\ Methods \\ Inclusion Criteria \\ Exclusion Criteria \\ Results \\ Samples \\ Quality of Studies \\ Study Designs \\ Interventions \\ Outcomes \\ Discussion \\ Limitations of the Studies \\ Limitations of the Review \\ Clinical/Practical Implications \\ Research Implications \\ Conclusions
}

BACKGROUND: Meditative movement, such as tai chi, yoga, and qi gong, may benefit people with cystic fibrosis (CF), as a form of gentle exercise incorporating meditation, breathing, and relaxation. Respiratory function is the most common issue in CF. In this systematic review we synthesized the evidence on the effect of meditative movement on respiratory function in patients with CF. METHODS: We searched Chinese and English language databases with terms relating to tai chi/yoga/qi gong, and respiratory function/cough/dyspnea. Articles were screened and selected by 2 researchers. We included controlled studies published in English or Chinese after 1980, and extracted data using a specially designed spreadsheet. Two researchers independently evaluated study quality and reporting, using 3 standardized checklists. Meta-analysis was not possible due to heterogeneous methods. RESULTS: We found 1,649 papers, included 43 (30 in English, 13 in Chinese), 23 of which were randomized controlled trials, and 20 were non-randomized trials. No studies were concerned with CF. Eleven studies included patients with respiratory disorders, and 27 included healthy people. Very few studies were high quality. The main problems with the randomized controlled trials was the randomization and non-random and/or poorly reported sampling. The main problems with the non-randomized studies were poor reporting of samples and non-equivalent groups. Although no clinically important changes were found, meditative movement may improve $\mathrm{FEV}_{1}$ in healthy people, compared to no treatment/exercise (the intervention groups showed effect-size changes from 0.07 to 0.83 ), but meditative movement did not appear to affect $\mathrm{FEV}_{1} / \mathrm{FVC}$ in subjects with COPD. Key study limitations were: poor reporting of sampling or methods; inadequate sample size; non-randomized design; inadequate description of randomization; randomization by center; no blinding; lack of reporting of important aspects of meditative movement; and short-term follow-up. CONCLUSIONS: The available evidence does not support meditative movement for patients with $\mathrm{CF}$, and there is very limited evidence for respiratory 
function in healthy populations. The available studies had heterogeneous populations and provided inadequate sampling information, so clinically relevant conclusions cannot be drawn. Well powered, randomized studies of meditative movement are needed. Key words: meditative movement; cystic fibrosis; tai chi; taichi; taiji; tai ji; yoga; qigong; qi gong; lung function; asthma; breathing exercises. [Respir Care 2014;59(3):427-440. (C) 2014 Daedalus Enterprises]

\section{Introduction}

This review was conducted to summarize the available evidence on meditative movement for respiratory function for clinical use and to inform research, in particular a feasibility study planned by the authors on the use of tai chi, a type of meditative movement, for adults with cystic fibrosis (CF). $\mathrm{CF}$ is a genetic disorder that particularly affects the respiratory and digestive systems. ${ }^{1} \mathrm{CF}$ is one of the United Kingdom's most common life-threatening inherited diseases, with over 9,000 people living with CF in the United Kingdom. ${ }^{1}$ The most common symptoms of $\mathrm{CF}$ are respiratory: persistent coughing and wheezing, and recurrent chest and lung infections, ${ }^{2}$ as well as poor weight gain, malabsorption, and malnutrition. ${ }^{3,4} \mathrm{CF}$ treatment varies between patients. However, common treatments in a pancreatic insufficient individual with chronic Pseudomonas aeruginosa infection may include airway clearance technique approximately twice a day; a high calorie diet with dietary supplements daily; pancreatic enzyme supplementation with all meals and snacks; at least one nebulized antibiotic twice a day; pulmozyme nebulized once a day; and azithromycin 3 times a week. ${ }^{5}$ They may also suffer stress, frustration, depression, irritability, worry, insomnia,/behavioral issues, and poor posture, and as a result miss school or work. ${ }^{4,6-8}$

Meditative movement is a recently coined term for gentle exercises that incorporate meditation, breathing, and relaxation. ${ }^{9}$ Meditative movement (also called complementary/alternative exercise or mind-body exercise) as a group of therapies is increasingly the focus of studies and re-

Dr Lorenc, Ms $\mathrm{Hu}, \mathrm{Mr}$ Mian, and Professor Robinson are affiliated with the Department of Allied Health Sciences, London South Bank University, London, United Kingdom. Ms Wang is affiliated with the Centre for Evidence-Based Chinese Medicine, Beijing University of Chinese Medicine, Beijing, China. Dr Madge is affiliated with the Department of Cystic Fibrosis, Royal Brompton Hospital, Sydney Street, London, United Kingdom.

This study was partly supported by the Tracie Lawlor Trust for Cystic Fibrosis. The authors have disclosed no conflicts of interest.

Correspondence: Ava B Lorenc PhD, Department of Allied Health, London South Bank University, 103 Borough Road, London SE1 0AA, United Kingdom. E-mail: lorenca@1sbu.ac.uk.

DOI: $10.4187 /$ respcare. 02570 views, with evidence identified for fibromyalgia ${ }^{10,11}$ and cognitive impairment. ${ }^{12}$ Meditative movement may be beneficial for people with $\mathrm{CF}$, as exercise is part of their recommended treatment package, ${ }^{5,13}$ although they may have limited functional capacity. However, a brief literature search identified that there was no specific research evidence on the use of meditative movement for patients with $\mathrm{CF}$. This review therefore focused on the effectiveness of meditative movement for respiratory function, which is the main clinical feature and cause of death in people with CF. ${ }^{14}$

In the United States, research indicates that $65 \%$ of children with CF use complementary and alternative medicine (including prayer), $49 \%$ being mind-body approaches. ${ }^{15}$ Complementary and alternative medicine has been used for $\mathrm{CF}$ symptoms such as mucus clearance, anxiety, and general health, with $77 \%$ finding it useful. ${ }^{15}$

The practice of meditative movement is popular in both China and the West. ${ }^{16-19}$ Meditative movement, most commonly tai chi, yoga, and qi gong, incorporates: focus of the mind; movements, usually slow, relaxed, flowing and choreographed; a focus on breathing to rest the mind, which also "energizes" the body; and a deep state of physical and mental relaxation. ${ }^{9}$ Tai chi and qi gong have ancient roots in China as martial practice, and yoga was originally a spiritual practice. ${ }^{20,21}$ Qi gong is considered the most ancient practice of bringing awareness to and directing the movement of "qi" in the body. ${ }^{20,22}$ Tai chi, which is one form of qi gong, is particularly choreographed and can be tailored to meet individual needs. ${ }^{20}$ Yoga similarly aims to create physical and emotional balance through the use of postures (asanas) and breathing exercises (pranayama). ${ }^{23}$ Tai chi and qi gong are based on the theory and philosophy of traditional Chinese medicine, in which "meridians" or energy channels throughout the body are used to treat different aspects of disease. Some meridians are related to organs in the body. The underpinning Chinese medical philosophy is that the lung meridian influences fluid metabolism and lung function. According to traditional Chinese medicine it is the expansive action of tai chi that affects the lungs and diaphragm and can also aid digestion and fluid movement in the body. ${ }^{24}$ In recent years evidence of positive effect for a variety of chronic conditions has emerged for all 3 practices, including cardiovascular disease, arthritis, and falls risk. ${ }^{16,21,25}$ 


\section{Meditative Movement for Respiratory Function: A Systematic Review}

A previous systematic review found positive evidence for people with asthma for improving cardiorespiratory function using yoga, although included studies were poor quality. ${ }^{26}$ Other reviews of tai chi for improving aerobic capacity, ${ }^{27}$ and yoga for improving pulmonary function, ${ }^{28}$ found positive evidence, but the focus was on healthy adults rather than patients with chronic disease, and the Chinese literature was not included. In addition, the focus was on tai chi as a form of aerobic exercise rather than a treatment for respiratory function. ${ }^{29}$ Although aerobic exercise is recommended for $\mathrm{CF},{ }^{13}$ excessive exercise can in some cases lead to dyspnea, stress, and fatigue. Conversely, meditative movement allows patients to work within their functional capacity, using gentle movements to stimulate movement of qi, mucus, blood, and lymph. Meditative movement's gentle yet demanding movements are lowimpact, low-stress, calm the sympathetic nervous system, and engage the parasympathetic system. ${ }^{24}$ This can improve cardiovascular and pulmonary function without release of stress hormones, aiding immune function, reducing inflammation due to chronic illness, preventing infections, and improving quality of life. ${ }^{24}$

This review synthesized the evidence available in Western and Chinese databases on meditative movement (tai chi, qi gong, or yoga) for respiratory function. Respiratory function was chosen as the main clinical feature since the primary cause of death in $\mathrm{CF}$ is lung disease. This review had 2 aims.

- Identify the gaps in research on meditative movement for respiratory function, to inform future research, in particular a trial of tai chi for adults with CF being conducted by the authors

- Summarize for CF clinicians and patients the evidence for meditative movement for respiratory function

Only controlled studies were included, with any control treatment, in any population. There was no protocol registered for this review.

\section{Methods}

We used the United States National Library of Medicine's Medical Subject Headings (MeSH) terms in the following search string: tai ji OR yoga OR breathing exercises AND cystic fibrosis OR dyspnea OR cough OR respiratory function tests OR respiration OR respiratory tract disorders OR breath tests. We used non-MeSH terms in the following search string: tai chi OR taichi OR taiji OR tai ji OR yoga OR qigong or qi gong AND cystic fibrosis OR respiratory system AND disorders/OR respiratory OR dyspnea OR cough OR respiration OR breath tests. To search the Chinese databases we used the search string: qigong OR yoga OR taiji AND cystic fibrosis OR lung function OR asthma OR dyspnea. An example search is given below.

1. Tai chi/

2. Breathing exercises/

3. Yoga/

4. 1 or 2 or 3

5. Cystic fibrosis/

6. Dyspnea/

7. Cough/

8. Respiratory function tests/

9. Respiration/

10. Respiratory tract disorders/

11. Breath tests/

12. 5 or 6 or 7 or 8 or 9 or 10 or 11

13. 4 and 12

The literature searches were carried out on English and Chinese language databases by native language speakers. The English databases, searched from their dates of conception until the end of 2011, were MEDLINE, all Cochrane Library resources, CINAHL, AMED, PsycINFO, ScienceDirect, and Index to Theses. The Chinese databases, searched from January 1, 1990, to April 1, 2012 were China National Knowledge Infrastructure, Vip, and Chinese BioMedical. The reference lists of included articles and all systematic reviews were also searched for additional references.

Articles were screened and selected by 2 researchers, based on the following study inclusion/exclusion criteria.

\section{Inclusion Criteria}

- Investigated tai chi, qi gong, or yoga

- Measured effects on CF or respiratory function, using one or more pulmonary function measurements or assessment scales: $\mathrm{FEV}_{1} / \mathrm{FVC}, \mathrm{FEV}_{1}$, maximum $\dot{\mathrm{V}}_{\mathrm{O}_{2}}$, Borg dyspnea scale, vital capacity, cardiorespiratory fitness index, peak expiratory flow

- Included in the English or Chinese databases

- Controlled trial

- Published later than 1980 , to ensure a certain level of quality

\section{Exclusion Criteria}

- Studied outcomes that do not measure pulmonary function (eg, ventilatory frequency, ventilatory equivalent, tidal volume, oxygen cost, respiratory pattern)

- Uncontrolled studies, letters, news items, reviews, case reports

- Published in 1980 or earlier

Data were extracted from each included article using a specially designed spreadsheet to collect information on 
Table 1. Criteria Used to Assign Quality Rating

\begin{tabular}{|c|c|c|c|}
\hline Randomized Controlled Trials & $\begin{array}{c}\text { CONSORT Items } \\
\text { Reported }\end{array}$ & $\begin{array}{l}\text { Cochrane Risk of } \\
\text { Bias Items Reported }\end{array}$ & $\begin{array}{c}\text { Meditative Movement } \\
\text { Reporting Checklist } \\
\text { Intervention Items Reported }\end{array}$ \\
\hline Very poor & Less than a third & 6 or more & Less than half \\
\hline Poor & Less than $60 \%$ & 5 and possible risk from 1 other & Between 40 and $60 \%$ \\
\hline Good & Between a third and $50 \%$ & 4 and possible risk from 1 other & More than half \\
\hline Very good & More than $60 \%$ & 3 or less from 1 other & More than $60 \%$ \\
\hline Non-randomized trials & $\begin{array}{l}\text { Trend Items } \\
\text { Reported }\end{array}$ & Quality Score & $\begin{array}{c}\text { Meditative Movement } \\
\text { Reporting Checklist } \\
\text { Intervention Items Reported }\end{array}$ \\
\hline Very poor & Less than a third & Less than $33 \%$ & Less than $25 \%$ \\
\hline Poor & Between 30 and $41 \%$ & Between 20 and $50 \%$ & Between 0 and $54 \%$ \\
\hline Good & Over 33\% & Between 37 and $100 \%$ & Over $29 \%$ \\
\hline
\end{tabular}

the intervention, control, outcomes, population, findings, and conclusions. Two researchers independently evaluated

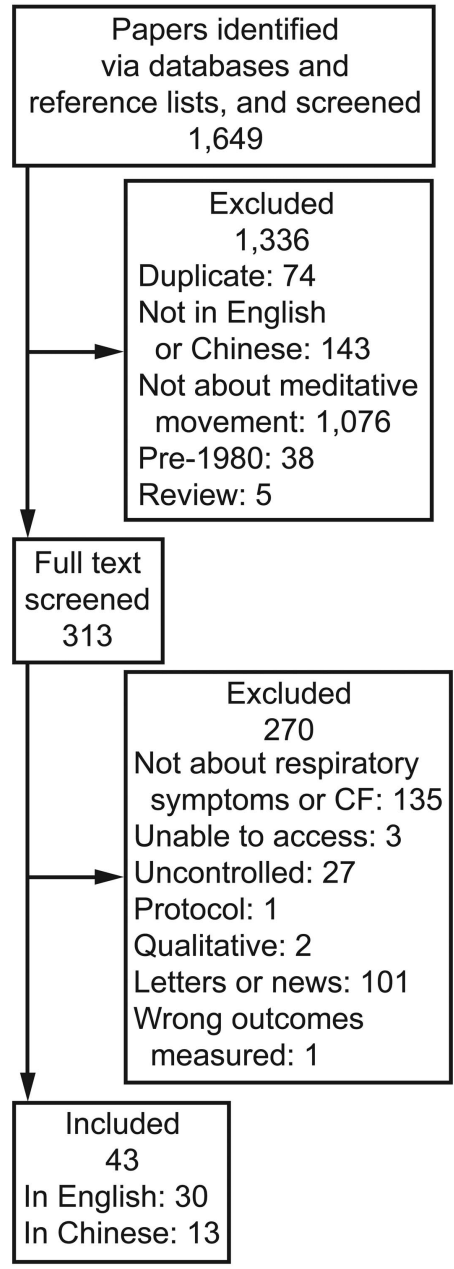

Fig. 1. Flow chart. $\mathrm{CF}=$ cystic fibrosis. the quality of the research and quality of the reporting, using 3 standardized checklists: for randomized controlled trials (RCTs) the Consolidated Standards of Reporting Trials (CONSORT) ${ }^{30}$ (plus extension for non-pharmacologic treatments, ${ }^{31}$ the Cochrane Risk of Bias assessment tool (http:// www.mrc-bsu.cam.ac.uk/cochrane/handbook/chapter_8/ table_8_5_a_the_cochrane_collaborations_tool_for_assess ing.htm), and the meditative movement reporting checklist. ${ }^{9}$ For non-randomized trials we used the Transparent Reporting of Evaluations with Nonrandomized Designs (TREND) statement checklist, ${ }^{32}$ the methods of Downs and Black, ${ }^{33}$ and the meditative movement reporting checklist. ${ }^{9}$

Each paper was given a score of 0 (not reported or high risk of bias), 0.5 (partially reported or possible risk of bias), or 1 (reported or no risk of bias) for each item on the checklist, summed to give total scores for reporting and quality (Table 1).

Given the extensive heterogeneity of interventions and comparison groups used in the studies, and the poor quality and reporting of many of the papers, a meta-analysis was deemed inappropriate and potentially misleading. Narrative synthesis was used for each combination of intervention and control, to describe the direction and size of effect and consistency across studies.

\section{Results}

We identified 1,632 papers: 889 in the English language databases and 743 in the Chinese databases. An additional 17 papers were identified from the reference lists. After excluding duplicates and studies with inappropriate study design or study focus, the final total of included studies was 43: 30 in English, 13 in Chinese (Fig. 1).

Twenty-three of the included studies were RCTs $\mathrm{s}^{34-55}$ and 20 were non-randomized trials. ${ }^{56-75}$ Of these, 13 eval- 


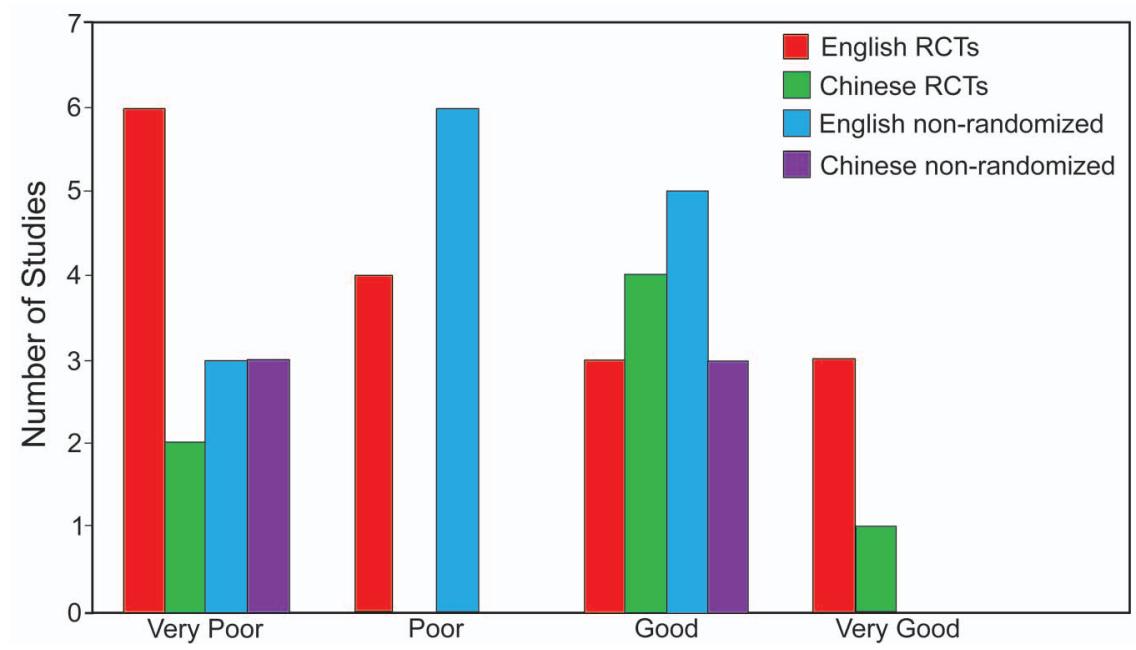

Fig. 2. Quality of included studies. RCT = randomized controlled trial.

uated yoga, 20 tai chi, 9 qi gong, and 1 tai chi and qi gong combined. Yoga, tai chi, and qi gong are discussed together in this review.

\section{Samples}

None of the studies identified included people with CF. Eleven studies focused on patients with respiratory disorders: COPD, , $8,41,43,45,51,52,72,75$ asthma ${ }^{42,47}$ and pleural effusion. ${ }^{48}$ Twenty-seven studied healthy people: older adults, ${ }^{34,35,50,55,57,58,60-65,73-77}$ healthy adults, ${ }^{59}$ healthy males, ${ }^{66,69}$ soldiers, ${ }^{44}$ students, ${ }^{46,53,71}$ children, ${ }^{37}$ "residents," ${ }^{59}$ and practitioners of tai chi/yoga. ${ }^{56,67,68}$ The remaining 6 studies were on non-respiratory disorders (lower-limb disabilities, ${ }^{70}$ breast cancer patients, ${ }^{54}$ heart disease, ${ }^{49}$ impaired vision, ${ }^{36}$ Parkinson's disease, ${ }^{40}$ and hypertension $^{39}$ ) but also measured respiratory outcomes. The majority of the studies involved adults; only 3 included children. $36,37,42$

The sample sizes ranged from 10 to 158 . Most studies had 20-60 subjects, and only 5 studies had over 100 .

\section{Quality of Studies}

As shown in Figure 2 and Tables 2 and 3, very few individual studies were rated very good (none of the nonrandomized studies, due to their inherent bias from using a non-randomized study design).

Figure 3 shows the change in the quality of studies over time; there was some improvement, but it is far from a constant trend.

The main risk of bias with RCTs was from problems with randomization (lack of reporting, or inadequate randomization process), and sampling (non-random or not reported, in particular no reporting about the population and how the sample was selected).

The main risks of bias in the non-randomized studies were due to lack of reporting of how and where the subjects were recruited, and non-equivalent groups. The nonrandomized trials had less variation in quality than did the RCTs, being overall poorer quality.

\section{Study Designs}

Thirty-eight studies were 2 -armed. These studies had control groups that received either no treatment or usual care (16 studies), or physical exercise (6 studies). Other controls included sedentary older people, matched controls, or another martial art, and 8 studies compared people experienced in meditative movement to people with no experience. Four studies were 3-armed, comparing meditative movement to exercise and to no intervention (2 studies); conventional treatment and physiotherapy; or yoga plus meditation or no intervention. One study was 4-armed.

\section{Interventions}

From the meditative movement checklist, ${ }^{9}$ most studies reported the duration and frequency of the intervention, and 59\% reported the style or philosophy. However, approximately two thirds of the studies did not adequately report details about the teacher, the teacher's qualifications or style, the degree of meditative focus, the degree of focus on breathing, achievement of deep relaxation (none reported), extent of practice at home, or results regarding adherence. The reporting of details of group composition, 


\section{Meditative Movement for Respiratory Function: A Systematic Review}

Table 2. Overall Quality of Studies

\begin{tabular}{|c|c|c|c|c|c|}
\hline & \multirow[b]{2}{*}{ Language } & \multicolumn{2}{|r|}{ Randomized Controlled Trials } & \multicolumn{2}{|r|}{ Not Randomized } \\
\hline & & Studies & Comments & Studies & Comments \\
\hline \multirow[t]{2}{*}{$\begin{array}{l}\text { Very } \\
\text { poor }\end{array}$} & English & $\mathrm{Six}^{36,42,45-48}$ & $\begin{array}{l}\text { Few or no details of how randomization or } \\
\text { blinding was performed. } \\
\text { Inadequate reporting of multiple factors. } \\
\text { Recruitment procedures not detailed and } \\
\text { no information on population or } \\
\text { generalizability of findings. } \\
\text { Some had small sample sizes. }\end{array}$ & Three $56,66,68$ & $\begin{array}{l}\text { No details of where the sample was recruited, } \\
\text { the population, or how the control group } \\
\text { was chosen. } \\
\text { Limited information on the intervention. } \\
\text { Small sample sizes. }\end{array}$ \\
\hline & Chinese & Two ${ }^{49,50}$ & $\begin{array}{l}\text { No details about the methods used to } \\
\text { generate the random allocation } \\
\text { sequence. } \\
\text { No information about how blinding was } \\
\text { done or who was blinded. } \\
\text { Recruitment procedures not detailed. } \\
\text { No information on how sample size was } \\
\text { determined. }\end{array}$ & Three $e^{71,72,75}$ & $\begin{array}{l}\text { Almost all factors not adequately reported. } \\
\text { Small sample size. }\end{array}$ \\
\hline Poor & English & Three $^{37,39,58}$ & $\begin{array}{l}\text { Either no information on randomization } \\
\text { procedure or bias in procedure (eg, } \\
\text { groups randomized rather than } \\
\text { individuals). } \\
\text { Did not report at least one important factor } \\
\text { (eg, control treatment, population, or } \\
\text { intervention). }\end{array}$ & $\operatorname{Six}^{61-64,67,69}$ & $\begin{array}{l}\text { Limited data on where the sample came from, } \\
\text { the population, or biased sampling (eg, } \\
\text { volunteer sample). } \\
\text { Limited information on the intervention. }\end{array}$ \\
\hline \multirow[t]{2}{*}{ Good } & English & Three $^{34,43,44}$ & $\begin{array}{l}\text { Although only one study had an adequate } \\
\text { description of randomization, the other } \\
\text { aspects of the studies were reported, } \\
\text { including detailed descriptions of the } \\
\text { interventions. }\end{array}$ & Five $^{35,59,60,65,70}$ & $\begin{array}{l}\text { No details of recruitment procedures. } \\
\text { Interventions and controls inadequately } \\
\text { described. }\end{array}$ \\
\hline & Chinese & Four ${ }^{52-55}$ & $\begin{array}{l}\text { No details about the randomization } \\
\text { methods. } \\
\text { The interventions and outcomes were } \\
\text { clearly described. }\end{array}$ & Three $^{52,73,74}$ & $\begin{array}{l}\text { Limited data on recruitment and bias risks } \\
\text { (eg, control group recruited at different } \\
\text { time and place). } \\
\text { Potential bias in comparison groups (eg, } \\
\text { non-equivalent groups or only a single } \\
\text { group). } \\
\text { Some description of intervention. }\end{array}$ \\
\hline \multirow[t]{2}{*}{$\begin{array}{l}\text { Very } \\
\text { good }\end{array}$} & English & Four $^{38,40,41,57}$ & $\begin{array}{l}\text { Randomization described in all studies. } \\
\text { Good descriptions of interventions. } \\
\text { Some blinding used. } \\
\text { Low risk of bias. }\end{array}$ & None & \\
\hline & Chinese & $\mathrm{One}^{52}$ & $\begin{array}{l}\text { Reported the randomization procedure. } \\
\text { Detailed description of inclusion criteria } \\
\text { and exclusion criteria. } \\
\text { Other aspects clearly reported. } \\
\text { Low risk of bias }\end{array}$ & None & \\
\hline
\end{tabular}

face to face or supporting materials, and types of movement varied widely, with around half reporting this information and a third not reporting any of these details.

The length of intervention ranged from 7 minutes to 2 hours; most common was 30-60 min. Yoga tended to be practiced for longer and more frequently than tai chi/ qi gong. Nine studies specified encouraging home practice, usually daily. Two used a video, one used a tape recording, one used a list of movements, and one used verbal instructions. Yang style tai chi was most common (6 studies). Others included "health" tai chi, simplified tai chi, Wu style tai chi, Chen style 32 tai chi, Cheng short style tai chi, light intensity tai chi, Shuxinpingxuegong (qi gong), Emei qi gong, Wu qinxi (qi gong), tai chi qi gong, qi gong, Iyengar yoga, Hatha yoga, Karlaripayattu yoga, and yoga respiratory training. Most studies used a 2 or 3 month follow-up period; a minority followed up for up to 5 years. 


\section{Meditative Movement for Respiratory Function: A Systematic Review}

\section{Outcomes}

As in previous systematic reviews, meta-analysis was not possible because the outcomes measured were so heterogeneous: less than half of the studies in any comparison group used the same outcome.

Table 3 shows the respiratory function outcome data. The results from studies assessed as very good and good suggest that:

- In healthy populations (including older people, soldiers, and students), meditative movement appears to improve $\mathrm{FEV}_{1}$, compared to no treatment or exercise, either daily or less frequently. The intervention groups showed effect-size changes of $0.07,{ }^{73} 0.31,{ }^{74} 0.6,{ }^{44}$ and $0.83 .{ }^{53}$ It is unlikely that meditative movement, compared with no treatment, affects $\dot{\mathrm{V}}_{\mathrm{O}}$, cardiorespiratory fitness index, or maximum aerobic capacity, although one study showed an increase of 0.23 (men) and 0.07 (women). ${ }^{64}$

- In people with COPD, meditative movement, compared to no treatment, does not appear to affect $\mathrm{FEV}_{1} / \mathrm{FVC}$. However, in 2 of these studies the intervention was less than weekly, ${ }^{38,43}$ which may have influenced outcomes. Meditative movement may improve $\mathrm{FEV}_{1} / \mathrm{FVC}$, compared to exercise, as found by Zhu et al, ${ }^{51}$ who also found a significant difference between 3 groups: meditative movement, exercise, and no treatment. However, $\mathrm{Xu}$ et $\mathrm{al}^{52}$ found that pulmonary rehabilitation was actually better than meditative movement.

- In people with Parkinson's disease, meditative movement may reduce the Borg dyspnea score by 1 point, compared to exercise, although only one study investigated that outcome. ${ }^{40}$

The studies rated as poor or very poor provide further evidence, although this is unreliable due to the study quality. These studies suggest that: weekly tai chi may improve maximum $\dot{\mathrm{V}}_{\mathrm{O}_{2}}$ in healthy older people, compared to sedentary older people (although from non-randomized studies, and all from the same research group $)^{61-64}$; weekly yoga may improve $\mathrm{FEV}_{1}$ in healthy people, including students, older people, and yogis, although this is based on matched rather than randomized controls, ${ }^{46,56,66}$ and vital capacity in older people..$^{50}$ In chronic-disease populations the poorer quality studies suggest that tai $\operatorname{chi}^{42}$ and yoga ${ }^{47}$ may improve respiratory function in asthma, and qi gong may improve respiratory function in cor pulmonale ${ }^{49}$ and COPD. ${ }^{72}$

\section{Discussion}

There is no published research on meditative movement and CF. In people with COPD, meditative movement, com- pared to no treatment, does not appear to affect $\mathrm{FEV}_{1} /$ FVC, although it may have an effect compared to exercise. The evidence on meditative movement for asthma is of poor quality. In people with Parkinson's disease, meditative movement may reduce the Borg dyspnea score by 1 point, compared to exercise, although that finding is from only one study. ${ }^{40}$

In healthy subjects there appears to be some preliminary good quality evidence, from 4 studies, ${ }^{44,53,73,74}$ that meditative movement improves $\mathrm{FEV}_{1}$, compared to either no treatment or exercise. The effect size range for $\mathrm{FEV}_{1}$ was $0.07-0.83$. This was confirmed by a meta-analysis of tai chi, which found improved aerobic capacity. ${ }^{27} \mathrm{~A}$ series of studies from one research group found evidence for tai chi improving $\dot{\mathrm{V}}_{\mathrm{O}_{2}}$ in healthy older people, but these studies were of poor quality. ${ }^{60,63-65}$ Yoga may improve $\mathrm{FEV}_{1}$, but the evidence is of poor quality. ${ }_{46,56,66}$

There is little evidence that meditative movement improves $\mathrm{FEV}_{1} / \mathrm{FVC}$. This may be due to these studies using a less frequent intervention ( 2 or 3 times a week). ${ }^{38,43,56,70}$ One study found that respiratory rehabilitation was better than meditative movement in improving $\mathrm{FEV}_{1} / \mathrm{FVC} .^{51}$ Meditative movement is unlikely to affect the cardiorespiratory fitness index, although only one study investigated the outcome. ${ }^{42}$ The results regarding clinical respiratory parameters may have implications for the design of future trials, because, although a commonly used outcome measure in clinical practice, these health parameters vary widely for people with CF; the broader impact of meditative movement on quality of life, well-being, and psychosocial outcomes may be more appropriate to capture in studies of meditative movement. 78,79

Although the studies included indicate that it is unlikely that meditative movement, compared with no treatment, affects $\dot{\mathrm{V}}_{\mathrm{O}_{2}}$ or maximum aerobic capacity, a previous metaanalysis of experimental studies of tai chi found a small effect size for $\dot{\mathrm{V}}_{\mathrm{O}_{2}}(0.33,95 \%$ CI $0.41-1.07){ }^{27}$

Most of the studies compared the intervention with either standard care or no treatment, which limits the interpretation of the evidence regarding the specific effects of meditative movement over and above extra attention. Many of the studies, especially those from Taiwan, compared experienced tai chi practitioners to sedentary people, which is a design with high risk of bias from confounding factors.

The details of the meditative movement used were heterogeneous, which made direct comparison problematic. Combination of results from high-quality studies did not reveal any definitive conclusions regarding the effective "dose" of meditative movement, or whether tai chi, yoga, or qi gong were more effective. Less than weekly practice did appear less likely to significantly improve spirometry. Although most studies reported the duration and frequency of intervention, there was very limited evidence, due to 


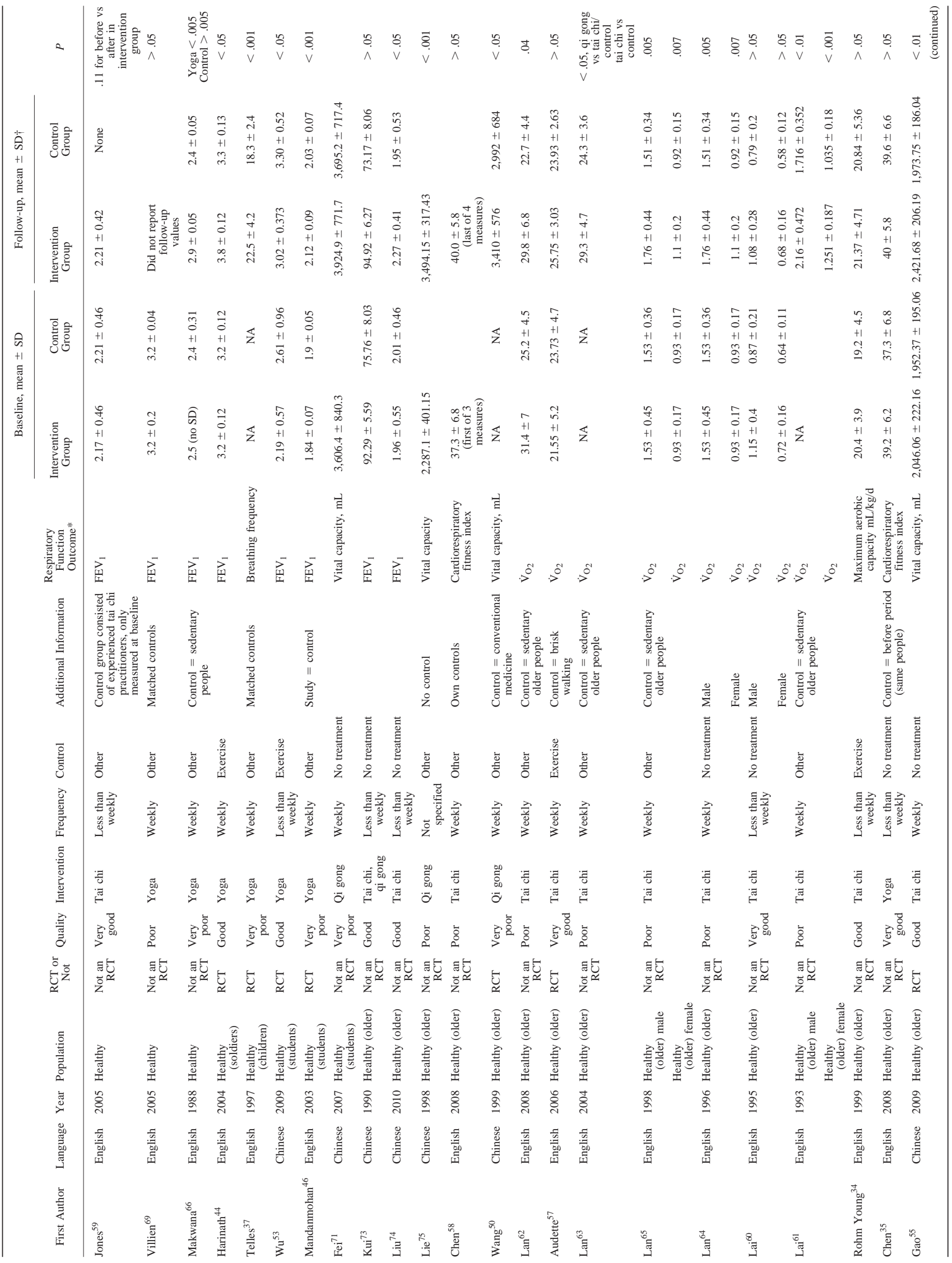




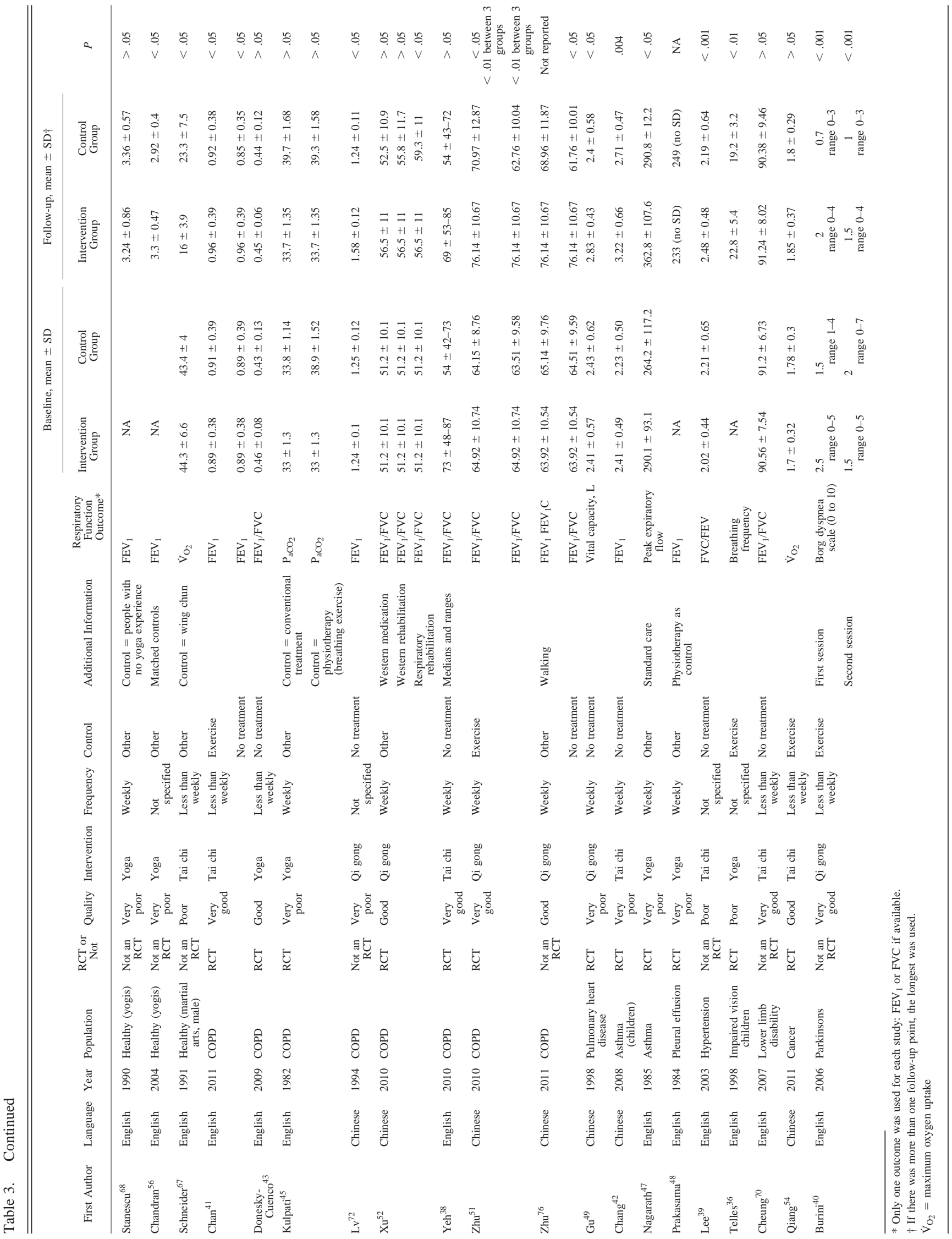




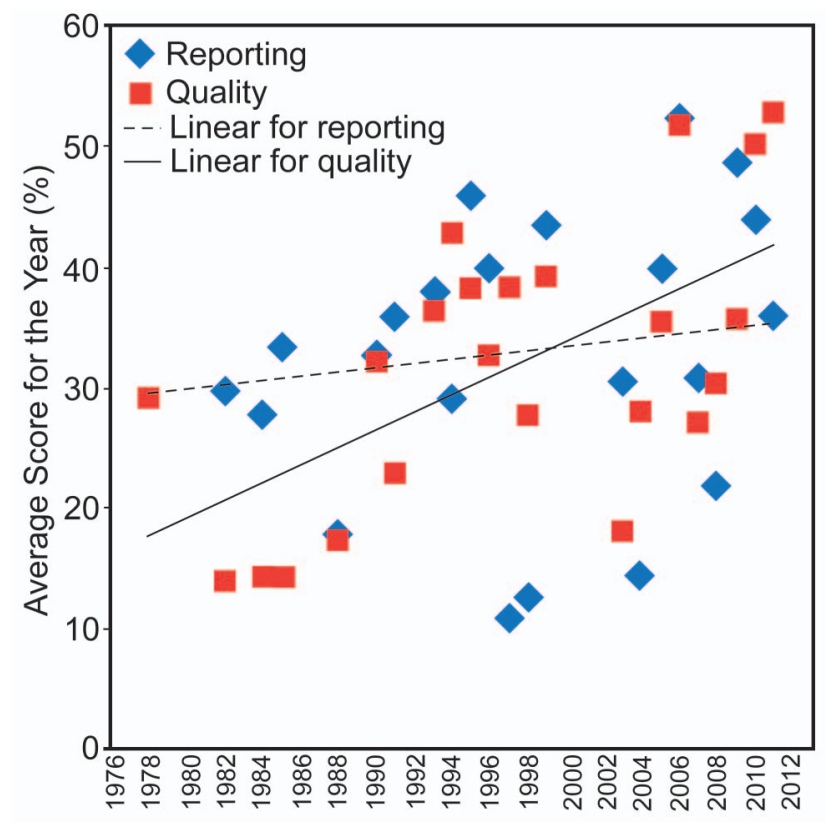

Fig. 3. Study quality versus time.

lack of reporting, for specific (important) aspects of meditative movement interventions. We are therefore unable to make firm conclusions regarding the details of the best approach to use as an intervention. Some studies suggested that meditative movement may act as a moderate intensity exercise, may stabilize the sympathetic nervous system/ condition autonomic function, decrease airway resistance, and improve muscle strength, but data are very limited for these outcomes.

\section{Limitations of the Studies}

Few studies were rated as very good. The main limitations of the studies are summarized below.

- Many studies did not report their sampling frame and sampling methods (ie, no information on how and where subjects were recruited). This problem severely limits study validity because sampling methods can introduce important bias and the results may not be generalizable.

- Many studies had small, potentially underpowered sample sizes.

- Non-random group allocation therefore means we cannot draw definitive conclusions as to the effect of the meditative movement, because non-randomized trials can be subject to confounding factors such as time-related or seasonal bias. Well conducted randomized trials are more likely to have internal validity and thus accurately estimate the causal effects of interventions than are nonrandomized studies.

- The randomization process was rarely described.

- Some studies randomized centers or schools rather than individuals, the implications of which were not adequately discussed.

- Blinding was rare, although blinding is challenging in complex interventions such as meditative movement. ${ }^{80}$

- The studies often did not include details about important aspects of the meditative movement, such as focus on meditative/breathing aspects, practice at home, and adherence, making it difficult to compare results and generalize in practice.

- Follow-up was usually a maximum of 3 months, which may be too short for those with chronic illnesses, who are likely to have long periods of ill health and fluctuations over time.

\section{Limitations of the Review}

- Although we accessed both English and Chinese language databases and papers, research in other languages may have been missed.

- Meta-analysis could not be performed due to the heterogeneity of outcomes.

- Dates were restricted to post-1980.

\section{Clinical/Practical Implications}

This review provides a summary of the current evidence for meditative movement and respiratory function, with implications for a CF population, although none of the studies included subjects with $\mathrm{CF}$. The findings relating to COPD suggest that meditative movement may not affect respiratory function, but this cannot be applied to a $\mathrm{CF}$ population. Very limited evidence from 4 studies suggests that meditative movement may improve $\mathrm{FEV}_{1}$ in healthy people. Although the application of these findings to $\mathrm{CF}$ patients is limited, they may be important, given the impact of declining lung function in $\mathrm{CF}$, and the common use of $\mathrm{FEV}_{1}$ as a clinical measure of lung function in $\mathrm{CF}$ (because it is quick, easy, and reflects air-flow limitation and lung volume). However, for CF patients, lung function alone as an end point is limited by the relative wellness of people with CF today, echoed by the low annual rate of decline in $\mathrm{FEV}_{1}\left(0.5 \%\right.$ per year) ${ }^{81}$ The impact of tai chi on aerobic capacity may also be affected by sex, exercise intensity, duration, frequency, and the subject's initial level of physical activity. ${ }^{27}$

This review has found that the evidence for meditative movement for people with $\mathrm{CF}$ is non-existent; however, 


\section{Meditative Movement for Respiratory Function: A Systematic Review}

evidence suggests that meditative movement has both physical and psychological benefits for people with chronic health conditions. ${ }^{78,79}$ The possible benefit of meditative movement for $\mathrm{CF}$ may not be respiratory function, but rather as a holistic intervention incorporating physical, psychological, social, and philosophical aspects, ${ }^{82}$ and in providing self-management, gentle exercise, flexibility, posture, mindfulness, and improved quality of life. Treatment for people with $\mathrm{CF}$ is complex and includes daily chest physiotherapy, exercise, supplements to avoid malnutrition, and oral and nebulized antibiotics (predominantly self-administered), resulting in high burden for patients. ${ }^{83}$ Most meditative movement can easily be tailored to suit individual needs and adapted for practice in a range of situations, including short time periods; standing, seated, or lying down positions; minimal space; without specialized materials or clothing; and in- or outdoors. Meditative movement may provide a very useful self-management tool for people with $\mathrm{CF}$, as an adjunct to conventional care. Meditative movement is also likely to improve flexibility and posture: a symptom and aggravator of CF. ${ }^{6}$ The mindful awareness of the movements can reduce the body's stress level and improve quality of life and well-being.

This review provides important implications for researchers to design more suitable studies with appropriate outcomes. It is difficult to apply the findings to existing meditative movement programs, given the huge variation in the definition of meditative movement, and lack of reporting of specific aspects (ie, Larkey's criteria ${ }^{9}$ ).

\section{Research Implications}

Due to the relative well-being of people with CF today, large numbers will be required for clinical trials to show any differences in lung function. The use of various patient reported outcomes should be included in addition to lung function, in order to gain a better understanding of the meaning of change in health status, which is individually relevant. Some of the studies in this review did include such measures. The importance of measuring objective physiological parameters has been emphasized by this review.

There is clearly a need for studies of meditative movement for people with $\mathrm{CF}$, as well as more studies on respiratory function in conditions other than COPD. The lack of research on meditative movement for CF may be a result of the emphasis in $\mathrm{CF}$ research on basic science and developing new life-prolonging medical treatments rather than complementary healthcare approaches. There is also a need to explore the broader impact of meditative movement on health and well-being, quality of life, and as a self-management tool, given the range of individual differences and lack of stability in day to day functioning in people with $\mathrm{CF}$.
The meditative movement interventions used in the included studies varied widely, in particular in duration, frequency, and support provision. Future research may wish to consider using a standardized intervention, using guidance $^{9}$ to report their intervention.

Given the limited evidence for meditative movement in this area, continuing to compare meditative movement to usual care, perhaps using comparative effectiveness studies, is more appropriate than prematurely attempting to explore specific effects or mechanisms through comparing to other forms of movement/exercise.

Future studies of meditative movement need to ensure the use of checklists such as CONSORT when designing studies. Many studies did not adequately report important details such as randomization processes, sampling, and intervention details. Reporting of meditative movement interventions according to Larkey' $s^{9}$ criteria was inadequate. Although duration and frequency is usually included, there is a need for reporting of other important aspects, namely details of the teacher, degree of meditative focus, degree of focus on breathing, achievement of deep relaxation, extent of practice at home, and adherence. This is important because the style of meditative movement may affect the results. ${ }^{27}$ Larkey suggests using methods such as brain wave activity (for level of relaxation) and specifically designed self-report measures (eg, for degree of meditative focus). ${ }^{9}$

Certain study designs are more appropriate for certain interventions and populations, ${ }^{84}$ and contention is emerging about how complementary medicine should be evaluated. ${ }^{85-90}$ The complexity of interventions such as meditative movement, including practitioner and non-specific effects, the influence of patient choice, and potential synergistic effects, require innovative evaluative approaches.

\section{Conclusions}

There is no evidence for meditative movement for CF, and some negative evidence in COPD, but results suggest that meditative movement may have the potential to improve respiratory function in healthy populations, although the evidence is very limited. Due to the heterogeneity of study populations and lack of information on sampling, clinically relevant conclusions cannot be drawn. More research is needed in this area, in particular on people with $\mathrm{CF}$, and well powered, randomized studies using broader outcomes such as quality of life and symptom scores.

\section{REFERENCES}

1. Cystic Fibrosis Trust. Annual data report 2010. http://www.cftrust. org.uk/aboutus/what_we_do/care/UK_CF_Registry_-_Annual_Data _Report_2010.pdf. Accessed January 7, 2014. 


\section{Meditative Movement for Respiratory Function: A Systematic Review}

2. NHS Choices. Symptoms of cystic fibrosis. 2012. http://www.nhs uk/Conditions/cystic-fibrosis/Pages/Symptoms.aspx. Accessed January 7, 2014.

3. Sawyer MG, Reynolds KE, Couper JJ, French DJ, Kennedy D, Martin $\mathrm{J}$, et al. Health-related quality of life of children and adolescents with chronic illness: a two year prospective study. Qual Life Res 2004;13(7):1309-1319.

4. Pfeffer PE, Pfeffer JM, Hodson ME. The psychosocial and psychiatric side of cystic fibrosis in adolescents and adults. J Cyst Fibros 2003;2(2):61-68.

5. Cystic Fibrosis Trust. The standards of care and good clinical practice for the physiotherapy management of cystic fibrosis. 2011. http:// www.cftrust.org.uk/aboutcf/publications/consensusdoc/ Physio_standards_of_care.pdf. Accessed January 7, 2014.

6. Goss CH, Edwards TC, Ramsey BW, Aitken ML, Patrick DL. Patient-reported respiratory symptoms in cystic fibrosis. J Cyst Fibros 2009;8(4):245-252.

7. Tattersall R, Walshaw MJ. Posture and cystic fibrosis. J R Soc Med 2003;96(Suppl 43):18-22.

8. Ward C, Massie J, Glazner J, Sheehan J, Canterford L, Armstrong D, et al. Problem behaviours and parenting in preschool children with cystic fibrosis. Arch Dis Child 2009;94(5):341-7.

9. Larkey L, Jahnke R, Etnier J, Gonzalez J. Meditative movement as a category of exercise: implications for research. J Phys Act Health 2009;6(2):230-238.

10. Mist SD, Firestone KA, Jones KD. Complementary and alternative exercise for fibromyalgia: a meta-analysis. J Pain 2013;6:247-260.

11. Langhorst J, Klose P, Dobos G, Bernardy K, Huñuser W. Efficacy and safety of meditative movement therapies in fibromyalgia syndrome: a systematic review and meta-analysis of randomized controlled trials. Rheumatol Int 2013;33(1):193-207.

12. Wu Y. Mind-body exercise and cognitive function: potential approaches to manage cognitive impairment- a meta-analysis. MA Thesis. Gerontology Theses 2012.

13. Philpott J, Houghton K, Luke A. Physical activity recommendations for children with specific chronic health conditions: Juvenile idiopathic arthritis, hemophilia, asthma and cystic fibrosis. Paediatr Child Health 2010;15(4):213-225.

14. Simmonds NJ. Ageing in cystic fibrosis and long-term survival. Paediatr Resp Rev 2013;14(Supplement 1):6-9.

15. Tanase A, Zanni R. The use of complementary and alternative medicine among pediatric cystic fibrosis patients. J Altern Complement Med 2008;14(10):1271-1273.

16. Jahnke R, Larkey L, Rogers C, Etnier J, Lin F. A comprehensive review of health benefits of Qigong and Tai Chi. Am J Health Promot 2010;24(6):e1-e25.

17. Zhang L, Layne C, Lowder T, Liu J. A review focused on the psychological effectiveness of Tai Chi on different populations. Evid Based Complement Alternat Med 2012;DOI: 10.1155/2012/678107.

18. Wang C, Bannuru R, Ramel J, Kupelnick B, Scott T, Schmid C. Tai Chi on psychological well-being: systematic review and meta-analysis. BMC CAM 2010;10(1):23.

19. Zhu W, Guan S, Yang Y. Clinical Implications of Tai Chi interventions: a review. Am J Lifestyle Med 2010;DOI: 10.1177/ 1559827610368777.

20. Rogers CE, Larkey LK, Keller C. A review of clinical trials of Tai Chi and Qigong in older adults. Western J Nurs Res 2009;31(2):245279.

21. Hart J. An overview of clinical applications of therapeutic yoga. Alt Compl Ther 2008;14(1):29-32.

22. Sancier K, Holman D. Multifaceted health benefits of medical Qigong. J Altern Complement Med 2004;10(1):163-166.

23. Gimbel MA. Yoga, meditation, and imagery: clinical applications. Nurse Pract Forum 1998;9(4):243-255.
24. Acton W. Eternal spring; Taijiquan, Qi gong, and the cultivation of health, happiness and longevity. London: Singing Dragon 2009.

25. Han A, Robinson V, Judd M, Taixiang W, Wells G, Tugwell P. Tai Chi for treating rheumatoid arthritis. Cochrane Database Syst Rev 2004;(3):CD004849.

26. Galantino ML, Galbavy R, Quinn L. Therapeutic effects of yoga for children: a systematic review of the literature. Pediat Phys Ther 2008;20(1):66-80.

27. Taylor-Piliae RE, Froelicher ES. The effectiveness of Tai Chi exercise in improving aerobic capacity: a meta-analysis. J Cardiovasc Nurs 2004;19(1):48-57.

28. Abel AN, Lloyd LK, Williams JS. The effects of regular yoga practice on pulmonary function in healthy individuals: a literature review. J Altern Complement Med 2012;19(3):185-190.

29. Taylor-Piliae RE, Froelicher ES. Measurement properties of Tai Chi exercise self-efficacy among ethnic Chinese with coronary heart disease risk factors: a pilot study. Eur J Cardiovasc Nurs 2004;3(4): 287-294.

30. Schulz K, Altman D, Moher D; CONSORT Group. CONSORT 2010 Statement: updated guidelines for reporting parallel group randomised trials. BMC Med 2010;8(1):18.

31. Boutron I, Moher D, Altman DG, Schulz KF, Ravaud P. Extending the CONSORT statement to randomized trials of nonpharmacologic treatment: explanation and elaboration. Ann Intern Med 2008;148(4): 295-309.

32. Des Jarlais DC, Lyles C, Crepaz N. Improving the reporting quality of nonrandomized evaluations of behavioral and public health interventions: the TREND statement. Am J Public Health 2004;94(3): 361-366.

33. Downs SH, Black N. The feasibility of creating a checklist for the assessment of the methodological quality both of randomised and non-randomised studies of health care interventions. J Epidemiol Community Health 1998;52(6):377-384.

34. Rohm Young D, Appel L, Jee S, Miller E. The effects of aerobic exercise and t'ai chi on blood pressure in older people: results of a randomised trial. J Am Geriatr Soc 1999;47(3):277-284.

35. Chen KM, Chen MH, Hong SM, Chao HC, Lin HS, Li CH. Physical fitness of older adults in senior activity centres after 24-week silver yoga exercises. J Clin Nurs 2008;17(19):2634-2646.

36. Telles S, Srinivas RB. Autonomic and respiratory measures in children with impaired vision following yoga and physical activity programs. Int J Rehabil Health 1998;4(2):117-122.

37. Telles S, Narendran S, Raghuraj P, Nagarathna R, Nagendra HR. Comparison of changes in autonomic and respiratory parameters of girls after yoga and games at a community home. Percept Mot Skills 1997;84(1):251-257.

38. Yeh GY, Roberts DH, Wayne PM, Davis RB, Quilty MT, Phillips RS. Tai Chi Exercise for Patients With Chronic Obstructive Pulmonary Disease: A Pilot Study. Resp Care 2010;55(11):1475-1482.

39. Lee MS, Lee MS, Choi ES, Chung HT. Effects of Qigong on blood pressure, blood pressure determinants and ventilatory function in middle-aged patients with essential hypertension. Am J Chinese Med 2003;31(3):489-497.

40. Burini D, Farabollini B, Iacucci S, Rimatori C, Riccardi G, Capecci $\mathrm{M}$, et al. A randomized controlled cross-over trial of aerobic training versus Qigong in advanced Parkinson's disease. Europa Medicophysica 2006;42(3):231-238.

41. Chan AW, Lee A, Suen LK, Tam WW. Tai chi Qigong improves lung functions and activity tolerance in COPD clients: a single blind, randomized controlled trial. Comp Ther Med 2011;19(1):3-11.

42. Chang YF, Yang YH, Chen CC, Chiang BL. Tai Chi Chuan training improves the pulmonary function of asthmatic children. J Microbiol Immunol Infect 2008;41(1):88-95. 


\section{Meditative Movement for Respiratory Function: A Systematic Review}

43. Donesky-Cuenco D, Nguyen HQ, Paul S, Carrieri-Kohlman V. Yoga therapy decreases dyspnea-related distress and improves functional performance in people with chronic obstructive pulmonary disease: a pilot study. J Altern Complem Med 2009;15(3):225-234.

44. Harinath K, Malhotra AS, Pal K, Prasad R, Kumar R, Kain TC, et al. Effects of Hatha yoga and Omkar meditation on cardiorespiratory performance, psychologic profile, and melatonin secretion. J Altern Complem Med 2004;10(2):261-268.

45. Kulpati DD, Kamath RK, Chauhan MR. The influence of physical conditioning by yogasanas and breathing exercises in patients of chronic obstructive lung disease. J Associ Physician I 1982;30(12):865-868.

46. Mandanmohan, Jatiya L, Udupa K, Bhavanani AB. Effect of yoga training on handgrip, respiratory pressures and pulmonary function. Indian J Physi Pharmacol 2003;47(4):387-392.

47. Nagarathna R, Nagendra HR. Yoga for bronchial asthma: a controlled study. BMJ Clin Res Ed 1985;291(6502):1077-1079.

48. Prakasamma M, Bhaduri A. A study of yoga as a nursing intervention in the care of patients with pleural effusion. J Adv Nurs 1984;9(2):127133.

49. Gu YH. Observation on the influence of health preserving qigong for cor pulmonale patients' lung function. Chinese Qigong 1998;2:12-13.

50. Wang XM. Recovery cure of chronic obstructive pulmonary diseases by Songjing and Respiratory Qigong in the environment of natural highly-densed air with anion. Mod Rehab 1999;3(8):914-915.

51. Zhu Y, Li N, Jin HZ. Effect on chronic obstructive pulmonary disease patient in stable condition by health Qigong-Wuqingxi exercise early treatment. J Liaoning Uni TCM 2010;12(6):107-109.

52. Xu YH, Wang JH, Yang FX, He XZ. The effects of respiratory rehabilitation training on pulmonary function and quality of life of patients with COPD. Chinese J Rehab 2010;25(2):120-122.

53. Wu JH, Zhao DS, Liu HP, Fu S. The effects of pulmonary function in female undergraduates after Hatha Yogic exercise. J Nanjing Institute Phys Edu Nat Sci 2009;8(3):38-40.

54. Qiang WM, Dong FQ, Yan L, Chen YH, Tang L. Comparison of two different exercise program in breast cancer patients after postoperative adjuvant chemotherapy. Chinese J Nurs 2011;46(6):537-540.

55. Gao YM, Yang WL. Effects of Taijishan on the old women in cardiopulmonary function and fat metabolism. J Hubei Sports Sci 2009;28(2):174-176.

56. Chandran CK, Nair RH, Shashidhar S. Respiratory functions in Kalaripayattu practitioners. Indian J Physi Pharmacol 2004;48(2):235-240.

57. Audette JF, Jin YS, Newcomer R, Stein L, Duncan G, Frontera WR. Tai Chi versus brisk walking in elderly women. Age Ageing 2006; 35(4):388-393.

58. Chen KM, Lin JN, Lin HS, Wu HC, Chen WT, Li CH, Kai Lo S. The effects of a simplified Tai-Chi exercise program STEP on the physical health of older adults living in long-term care facilities: a single group design with multiple time points. Int J Nurs Stud 2008;45(4): 501-507.

59. Jones AY, Dean E, Scudds RJ. Effectiveness of a community-based Tai Chi program and implications for public health initiatives. Arch Phys Med Rehab 2005;86(4):619-625.

60. Lai JS, Lan C, Wong MK, Teng SH. Two-year trends in cardiorespiratory function among older Tai Chi Chuan practitioners and sedentary subjects. J Am Geriatr Soc 1995;43(11):1222-1227.

61. Lai JS, Wong MK, Lan C, Chong CK, Lien IN. Cardiorespiratory responses of Tai Chi Chuan practitioners and sedentary subjects during cycle ergometry. J Formos Med Assoc 1993;92(10):894-899.

62. Lan C, Chen S, Lai J. Changes of aerobic capacity, fat ratio and flexibility in older TCC practitioners: a five-year follow-up. Am J Chinese Med 2008;36(6):1041-1050.

63. Lan C, Chou S, Chen S, Lai J, Wong M. The aerobic capacity and ventilatory efficiency during exercise in Qigong and Tai Chi Chuan practitioners. Am J Chinese Med 2004;32(1):141-150.
64. Lan C, Lai J, Wong M, Yu M. Cardiorespiratory function, flexibility, and body composition among geriatric Tai Chi Chuan practitioners. Arch Phys Med Rehab 1996;77(6):612-616.

65. Lan C, Lai JS, Chen SY, Wong MK. 12-month Tai Chi training in the elderly: its effect on health fitness. Med Sci Sport Exer 1998; 30(3):345-351.

66. Makwana K, Khirwadkar N, Gupta HC. Effect of short term yoga practice on ventilatory function tests. Indian J Physiol Pharmacol 1988;32(3):202-208

67. Schneider D, Leung R. Metabolic and Cardiorespiratory Responses to the Performance of Wing Chun and T'ai Chi Chuan Exercise. Int J Sports Med 1991;12(03):319-323.

68. Stânescu DC, Nemery B, Veriter C, Marchal C. Pattern of breathing and ventilatory response to $\mathrm{CO}_{2}$ in subjects practicing hatha-yoga. J Appl Physiol 1981;51(6):1625-1629.

69. Villien F, Yu M, Bartholomy P, Jammes Y. Training to yoga respiration selectively increases respiratory sensation in healthy man. Resp Phsuiol Neurobi 2005;146(1):85-96.

70. Cheung SY, Tsai E, Fung L, Ng J. Physical benefits of Tai Chi Chuan for individuals with lower-limb disabilities. Occup Ther Int 2007;14(1):1-10.

71. Fei HC, Jin XK, Wang H. Research on the influence of "health qigong- six letters formula" to college students' cardiopulmonary function. J Jilin Institute Physical Educ 2012;23(2):67-68.

72. Lv ZM, Huang YZ, Gao CG, Chen N, Hong Q, Li LJ. Comparison of the effect of Emei Qigong on lung function for treatment of different types of chronic obstructive pulmonary diseases. J Luzhou Med Coll 1994;17(3):197-200.

73. Kui RQ, Lin YH, Sun YX, Zhou N. The effect of Qigong and Taijiquan on pulmonary function in the elderly. Chinese J Rehab 1990;5(3):115-117.

74. Liu XD, Jin HZ. Observation on effect of Taiji Chuan on cardiopulmonary function of older people. China Prsc Med 2010;5(2):34-35.

75. Lie WS. The influence of Qigong on lung function, sleep and mood of old men. J Luzhou Med Coll 1998;21(5):388-389.

76. Zhu Z. Health Qigong's Effect on the 61 stabilized patients with chronic obstructive pulmonary disease. J Nanjing TCM Uni 2011; $5(3): 235-7$

77. Li XH. Effect of Taijiquan exercise on lung function of old men. J Henan Normal Uni Nat Sci 2008;36(3):123-125.

78. Ng BHP, Tsang HWH. Psychophysiological outcomes of health qigong for chronic conditions: a systematic review. Psychophysiology 2009;46(2):257-269.

79. Wang C, Collett J, Lau J. The effect of tai chi on health outcomes in patients with chronic conditions: a systematic review. Arch Intern Med 2004;164:2503-2504.

80. Mason S, Tovey P, Long AF. Evaluating complementary medicine: methodological challenges of randomised controlled trials. BMJ 2002; 325(7368):832-834.

81. Taylor-Robinson D, Whitehead M, Diderichsen F, Olesen HV, Pressler T, Smyth RL, Diggle P. Understanding the natural progression in $\% \mathrm{FEV}_{1}$ decline in patients with cystic fibrosis: a longitudinal study. Thorax 2012;67(10:860-866

82. Wayne PM, Fuerst ML. The Harvard Medical School guide to Tai Chi: 12 weeks to a healthy body, strong heart, and sharp mind. London: Harvard Health; 2013.

83. Myers LB. An exploratory study investigating factors associated with adherence to chest physiotherapy and exercise in adults with cystic fibrosis. J Cyst Fibros 2009;8(6):425-7.

84. University of York Centre for Reviews and Dissemination. CRD's guidance for undertaking reviews in health care. 2008. http://www.york. ac.uk/inst/crd/pdf/Systematic_Reviews.pdf Accessed January 6, 2014. 


\section{Meditative Movement for Respiratory Function: A Systematic Review}

85. Walker LG, Anderson J. Testing complementary and alternative therapies within a research protocol. Eur J Cancer 1999;35(11):16141618.

86. Walji R, Boon H. Redefining the randomized controlled trial in the context of acupuncture research. Complement Ther Clin Pract 2006; 12(2):91-96.

87. Shea JL. Applying evidence-based medicine to traditional Chinese medicine: debate and strategy. J Altern Complement Med 2006; 12(3):255-263.
88. Herman PM, D'Huyvetter K, Mohler MJ. Are health services research methods a match for CAM? Altern Ther Health Med 2006; 12(3):78-83.

89. Broom A. Using qualitative interviews in CAM research: A guide to study design, data collection and data analysis. Complement Ther Med 2005;13(1):65-73.

90. Walach H, Falkenberg T, Fonnebo V, Lewith G, Jonas W. Circular instead of hierarchical: methodological principles for the evaluation of complex interventions. BMC Med Res Methodol 2006;6(1):29. 